INTEGRATING MODELING, ALGORITHM DESIGN, AND COMPUTATIONAL IMPLEMENTATION TO SOLVE A LARGE-SCALE NONLINEAR MIXED INTEGER PROGRAMMING PROBLEM ${ }^{\dagger}$

\title{
F. GLOVER
}

Department of Management Science, University of Colorado, Boulder, CO 80302, USA

D. KLINGMAN

David Bruton, Jr., Centennial Chain in Business Decision Support Systems, University of Texas at Austin, Austin, TX 78712, USA

\section{N. PHILLIPS}

Department of Operations Research, University of Texas at Austin, Austin, TX 78712, USA

and

\section{G.T. ROSS}

Production and Distribution Planning Systems, Analysis, Research, and Computation, Inc., Austin, TX 78767, USA

\begin{abstract}
This paper describes the formulation of a nonlinear mixed integer programming model for a large-scale product development and distribution problem and the design and computational implementation of a special purpose algorithm to solve the model. The results described demonstrate that integrating the art of modeling with the sciences of solution methodology and computer implementation provides a powerful approach for attacking difficult problems. The efforts described here were successful because they capitalized on the wealth of existing modeling technology' and algorithm technology, the availability of efficient and reliable optimization, matrix generation and graphics software, and the speed of large-scale computer hardware. The model permitted the combined use of decomposition, general linear programming and network optimization within a branch and bound algorithm to
\end{abstract}

${ }^{\dagger}$ This research was supported in part by the Office of Naval Research Contract N00014-78-C-0222, by the Center for Business Decision Analysis*, by the University of Texas at Austin, and by the David Bruton, Jr., Centennial Chair in Business Decision Support Systems. Reproduction in whole or in part is permitted for any purpose of the U.S. Government.

${ }^{\star}$ Center for Business Decision Analysis, Graduate School of Business - GSB 3.126, University of Texas, Austin, Texas 78712, USA.

๑ J.C. Baltzer A.G., Scientific Publishing Company 
overcome mathematical complexity. The computer system reliably found solutions with considerably better objective function values 30 to 50 times faster than had been achieved using general purpose optimization software alone. Throughout twenty months of daily use, the system was credited with providing insights and suggesting strategies that led to very large dollar savings.

\section{Keywords and phrases}

Nonlinear programming, decomposition, branch and bound, network, transportation, mixed integer programming.

\section{Authors' note}

A confidentiality agreement between the authors and the firm facing the problem described in the paper prohibits disclosure of the firm's name and operational or financial information relating to this application. We have, therefore, camouflaged a number of details concerning the business problem. The mathematical model, the algorithm and the key aspects of the implementation, however, remain intact.

\section{Introduction}

The need to maximize expected future revenue under complex contractual terms provided the impetus to develop a mixed integer nonlinear mathematical programming model and to implement a customized algorithm for its solution. The context which gave rise to the model is described here, although specifics of the actual case are disguised. How the structure of the model was exploited by a branch and bound algorithm that made combined use of the general purpose linear programming optimizer and a general purpose network optimizer is also discussed.

The technical accomplishments reported here are several.

First, the solutions provided by the model were high quality along several dimensions. In formal terms, they were provably 90-95\% optimal. In relative terms, they were at least $20 \%$ better than any the company had devised previously, and this savings amounted to a very large sum of money. In functional terms, they were relied upon by management in making operating decisions over a period of twenty months.

Second, the computational performance of the algorithm is noteworthy, given the scale and complexity of the problem. Early attempts by the company to optimize other formulations with general purpose algorithms had faired poorly. After eight cpu 
hours of time on an IBM 3033 computer, IBM's general purpose mixed integer programming system MPSX/370-MIP had found only a single feasible solution known to be only $75 \%$ optimal. (However, as discussed further below, the superiority of this suboptimal solution over the best solution derived by manual efforts convinced management of the value of an optimization model and spawned the project described here.) In contrast, the special purpose procedure described below routinely found an incumbent solution at the initial node of the branch and bound tree and verified 90-95\% optimal solutions in $1 / 2$ to $1 \mathrm{cpu}$ hour. Typical problems had 8500 linear constraints, 100 nonlinear constraints, 12000 continuous variables and 110 to 200 integer variables.

Third, the features of the algorithm show how optimization methodology and available optimization software tools may be combined to overcome problem complexity. Insights concerning exploitable problem structure which gave rise to the algorithm and which made the problem tractable are discussed in detail. The key features of the algorithm are decomposition (facilitated by replacing a large number of constraints by a very few constraints), relaxation and restriction (obtained by replacing nonlinear equations by linear ones), and network optimization (permitted by the decomposition).

The software implementation of the algorithm was carried out by Analysis, Research and Computation, Inc. (ARC). It involved IBM's general purpose linear programming system MPSX/370 [4], ARC's primal network optimization software ARCNET [1], a custom branch and bound routine written in FORTRAN which called both MPSX/370 and ARCNET as subroutines, and interface routines written in IBM's extended control language (ECL). Further details are given later in the paper.

Computer graphics were also instrumental in the successful use of the model. Two graphs, originally designed by the user as a mechanism for manually computing a solution, were subsequently plotted from the model solution. The graphs summarized in a comprehensive and clear fashion the results of an optimization run. These graphs were helpful both in revealing the implications of a solution and in identifying anomalies in solutions when the system was being debugged and refined.

From a broader perspective, the success of this effort illustrates two important points about the state-of-the-art of mathematical prograraming. Foremost, it gives evidence of the value of optimization methodology in practical applications. Prior to building the model described here, the company had formed a large task force of various professionals (managers, engineers, lawyers, economists and system analysts) to explore alternatives and to develop recommendations for resolving the business issues. Some of these persons spent considerable amounts of time developing specific strategies by hand (in essence, attempting to solve the model by hand). For their timeconsuming and tedious efforts, they produced answers which were technically infeasible and, without assigning a penalty for infeasibility, substantially inferior from 
a revenue perspective to the suboptimal solution produced by an early mathematical programming model. Thus, while the initial mathematical programming model developed for this application was impractical to use from a computer run-time viewpoint, it was very successful in demonstrating the potential of computer-based optimization. A very conservative estimate of the benefit cost ratio for this project is 500 to 1 .

In the second place, the success of the specialized algorithms demonstrates that mathematical programmers have developed robust modeling, solutions and implementation technologies. Although problems may be difficult to solve by general purpose algorithms and software, highly specialized approaches that integrate model, algorithm and computer implementation are viable.

\section{Problem description and mathematical model}

The $\mathrm{ABC}$ Corporation, as we shall refer to the company that faced the problem, was interested in establishing a five-year monthly operating plan for introducing new products and determining product distribution to customers consistent with its standing contracts, technological limitations, and governmental regulations. ABC operated in an environment characterized by volatile selling prices and changing governmental regulations which forced it to exercise care in establishing and fulfilling its obligations to customers.

The ABC Corporation had three types of customers which we will refer to by the collective designations of customer 1,2, and 3. Customer 3 was a 'standard' customer group who paid the prevailing market price for $\mathrm{ABC}$ products. For customers 1 and 2, all of $A B C$ 's products were interchangeable, and they had negotiated long-term contracts which assured them access to products, independent of product type, at a price considerably below the prevailing market price of ABC's least expensive product. More precisely, according to the contractual agreement, customer 1 was entitled to receive a fixed total amount of product over the life of the agreement. This amount was provided to customer 1 at a fairly stable supply rate by month. Customer 2, likewise, was entitled to purchase a fixed amount of product at a low price and at a steady supply rate until a particular moment in time, called the critical date. Thereafter, customer 2 purchased those product types which he was supplied during a critical period at a higher price but below the market price. (The critical period was a period of time immediately preceding the critical date.) Except for a provision to be discussed, customer 2 had the right to purchase after the critical date all the remaining supply of those product types sold to him during the critical period. ABC's production of each product type was heavily dependent on the availability of certain depletable inputs and thus, annual production levels of each product type normally diminished over time.

One further proviso of the agreement complicated ABC's arrangement with customers 1 and 2. After the critical date, $\mathrm{ABC}$ could, at its option, deliver to customer 
1 a portion of the supply of products to which customer 2 is entitled. The stipulation was that customer 1 could be sold no greater percentage of the remaining supply of a product type than the share he had received of the total amount of that product provided to customers 1 and 2 during the critical period. For example, suppose 105 units of a certain type were available during the critical period, 90 of which were sold to customer 3,10 to customer 2 , and 5 to customer 1 . Then after the critical period, customer 1 could receive up to $1 / 3$ of the product type available $(1 / 3=5 /(5+10))$, customer 2 would receive at least $2 / 3(10 /(5+10))$ of the product type available, and customer 3 would receive none. Further, no matter how small the amount of a particular type of product was used to supply customer 2 during the critical period, his rights to all of the remaining supply were assured.

Additional complications arose out of governmental regulations. In essence, $\mathrm{ABC}$ 's products may be conceived as coming in two major classes, Orange and White. Government policy dictated that during any calendar year, not more than one-half of the commodity delivered to customer 1 could be white.

The mathematical formulation that expresses the foregoing conditions follows. A detailed explanation of the problem notation follows the mathematical statement.

$$
\begin{array}{ll}
\text { Maximize } & \sum_{t \in T} \sum_{i \in I} \sum_{i \in J} c_{i j t} x_{i j t}-\sum_{i \in I_{\mathrm{N}}} e_{i} y_{i} \\
\text { subject to } \quad & \sum_{i \in I} x_{i j t}=d_{j t} \text { for } j=1,2, t \in T_{\mathrm{B}} \\
& \sum_{j \in J} x_{i j t}=a_{i t} \quad \text { for } i \in I_{\mathrm{E}}, \quad t \in T_{\mathrm{B}} \\
& \sum_{j \in J} x_{i j t}=a_{i t} y_{i} \text { for } i \in I_{\mathrm{N}}, \quad t \in T_{\mathrm{B}} \\
& \sum_{i \in I_{\mathrm{W}}} \sum_{t \in T_{k}} x_{i 1 t} \leqslant 0.5 \sum_{t \in T_{k}} d_{1 t} \text { for } k \text { such that } T_{k} \text { in } T \\
& x_{i 2 t} \leqslant a_{i t} z_{i} \text { for } i \in I, t \in T_{\mathrm{c}} \\
& \frac{\sum_{t \in T_{\mathrm{c}}} x_{i 2 t}\left(x_{i 1 t}+x_{i 2 t}\right)}{x_{t \in T_{\mathrm{c}}}}=q_{i} \text { for } i \in I
\end{array}
$$




$$
\begin{aligned}
& \sum_{i \in I} x_{i 1 t}=d_{1 t} \quad \text { for } t \in T_{\mathrm{A}} \\
& \sum_{j \in J} x_{i j t}=a_{i t} \quad \text { for } i \in I_{\mathrm{E}}, \quad t \in T_{\mathrm{A}} \\
& \sum_{i \in J} x_{i j t}=a_{i t} y_{i} \text { for } i \in I_{\mathrm{N}}, \quad t \in T_{\mathrm{A}} \\
& x_{i 1 t} \leqslant a_{i t}\left(1-q_{i}\right) \text { for } i \in I, \quad t \in T_{\mathrm{A}} \\
& x_{i 3 t} \leqslant a_{i t}\left(1-z_{i}\right) \text { for } i \in I, \quad t \in T_{\mathrm{A}} \\
& \epsilon z_{i} \leqslant q_{i} \leqslant z_{i} \quad \text { for } i \in I \\
& z_{i} \leqslant y_{i} \quad \text { for } i \in I_{\mathrm{N}} \\
& 0 \leqslant z_{i} \leqslant 1 \quad \text { and integer for } i \in I \\
& 0 \leqslant y_{i} \leqslant 1 \quad \text { and integer for } i \in I_{\mathrm{N}} \\
& x_{i j t} \geqslant 0 \quad \text { for } i \in I, \quad j \in J, t \in T,
\end{aligned}
$$

where

I denotes the set of all product types,

$I_{\mathrm{N}} \quad$ denotes the set of new types of product,

$I_{\mathrm{E}} \quad$ denotes the set of existing types of product,

$I_{\mathrm{W}}$ denotes the set of product types belonging to the white class,

$J$ denotes the set of customer classes,

$T$ denotes the set of months in the time horizon,

$T_{\mathrm{c}} \quad$ denotes the set of months in the critical period in $T$,

$T_{k} \quad$ denotes the set of months in the $k$ th calendar year in $T$,

$T_{\mathrm{B}} \quad$ denotes the set of months before the critical date (includes $T_{\mathrm{c}}$ ),

$T_{\mathrm{A}} \quad$ denotes the set of months after the critical date,

$x_{i j t} \equiv$ units of the product type $i$ sold to customer $j$ in month $t$, 


$$
\begin{aligned}
& z_{i} \equiv\left\{\begin{array}{l}
1 \text { if type } i \text { is used to supply customer } 2 \text { during the critical period } \\
0 \text { otherwise }
\end{array}\right. \\
& q_{i} \equiv \begin{array}{l}
\text { portion of the amount delivered of type } i \text { to customer } 2 \text { of the total } \\
\text { delivered to customers } 1 \text { and } 2 \text { during the critical period, }
\end{array} \\
& y_{i} \equiv\left\{\begin{array}{l}
1 \text { if new type } i \text { product is acquired } \\
0 \text { otherwise }
\end{array}\right. \\
& a_{i t} \equiv \text { forecasted supply of type } i \text { in month } t, \\
& d_{j t} \equiv \text { expected demand of customer } j \text { in month } t, \\
& c_{i j t} \equiv \text { discounted net present value of gross margin (selling price-delivered } \\
& e_{i} \equiv \text { investment pert required to acquire product of type } i, \\
& \epsilon \equiv \text { very small positive value to assure that } q_{i} \text { is positive if } z_{i} \text { is one. }
\end{aligned}
$$

The foregoing mathematical representation is admittedly somewhat complex. However, a brief descriptive breakdown of its components will help to clarify the model structure and make its features more understandable. The following remarks should be interpreted relative to the verbal description of the problem that preceded the formulation.

The objective function (1) measures the total discounted net present value of future revenues associated with sales to all customers, less any cost associated with the acquisition of new types of products. The first few constraints are chiefly 'technological' constraints affecting the critical period. Constraints (2) ensure that customers 1 and 2 will receive their appropriate known amounts during the time periods preceding the critical date. Constraints (3) and (4) ensure that a product of a given type delivered to customers equals the amount available in each time period before the critical date. Constraints (4) also reflect the impact of deciding not to acquire a new product type.

The next several constraints have to do with regulatory and contractual considerations. Constraints (5) ensure meeting the governmental regulation limiting delivery of white product to customer 1 during any calendar year of the model horizon. Constraints (6) and (7) are best understood when considered in conjunction with constraints (11) and (12). Constraints (11) reflect that the maximum share of the supply of a product type that can be sold to customer 1 is one minus the share that must go to customer 2. Constraints (12) preclude any sales to customer 3 after the critical date of those types used to supply customer 2 during the critical period.

Constraints (8), (9) and (10) perform the same role in the model for time periods after the critical date as constraints (2), (3) and (4) do for periods before 
the critical date. Note, however, that there is fixed demand only for customer 1, which will be important in the decomposition decsribed below. Constraints (13) through (17) express basic relationships needed for logical consistency.

The constraints that pose the greatest mathematical complexity are those of (7), each of which is nonlinear and has a point of discontinuity. These constraints, the large problem size, and the presence of integer variables, combine to make the problem exceedingly difficult to solve by any standard measure. In fact, the size of the problem itself is formidable. Depending upon the particular scenario management needed to analyze, a typical model comprised 40 time periods, $70-130$ types of existing products, and 5 to 10 types of new products. This resulted in models with 7000 to 8500 constraints (not counting simple upper bounds on variables) and 12000 continuous variables. The number of discrete $z_{i}$ variables (and associated $q_{i}$ variables) varied between 60 and 200 depending upon the scenario, with 100 to 130 being typical. This meant that there were normally 100 to 130 constraints of the form (7) in the model.

\section{Solving the model}

When we were confronted with this problem, there was no existing algorithm that could reasonably be expected to solve the model as specified. Most nonlinear programming algorithms require that nonlinear functions be differentiable and the combination of non-differentiability and integer variables puts the problem beyond the scope of those algorithms $[3,5,6]$. Other formulations had been considered by $\mathrm{ABC}$, including variants which allowed $q_{i}$ to take on discrete values (e.g. 0.05, 0.10, 0.15 ), but they increased the number of constraints and the number of integer variables. Attempts were made by $\mathrm{ABC}$ to solve one such model, but it proved exceedingly difficult to optimize. IBM's system for mixed integer linear programming MPSX/370MIP required eight cpu hours on an IBM 3033 computer to find a single integer solution, and this could only be proven to be within $25 \%$ of optimality. Next, ABC tried Beale's separable programming approach, without success [2] .

In the context of a branch and bound method for handling the discrete variables, the structure of the model and the terms of the contract itself suggested that a decomposition approach be used to obtain tractable subproblems to solve at each step. The natural idea is to segregate the problem into two major components, creating one model for decisions up through the critical period and a second model for time periods following the critical date. The naturalness of this approach was reinforced by the fact that the number of periods before ( 19 periods) and after ( 21 periods) the critical date were close to the same.

However, an additional more powerful strategy was required to make this type of decomposition effective. Assume for the moment that no constraint of (5) includes variables from both $t \in T_{\mathrm{B}}$ and $t \in T_{\mathrm{A}}$. (This assumption is incorrect but 
can be overcome, as will be shown.) Then it follows that the model decomposes into one covering $T_{\mathrm{B}}$ and one covering $T_{\mathrm{A}}$. The $T_{\mathrm{B}}$ model includes constraints (2)-(7) and (13)-(17). The $T_{\mathrm{A}}$ model includes constraints (8)-(12) and (17). Constraints (17) appear in both models, as do the relevant portions of the objective function. Disregarding the fact that the $T_{\mathrm{B}}$ model is a difficult nonlinear mixed integer problem, solving it would yield values for $z_{i}$ and $q_{i}$ to use in the $T_{\mathrm{A}}$ model. However, solutions to the $T_{\mathrm{B}}$ model might provide terribly bad values for $z_{i}$ and $q_{i}$ because they do not appear in the $T_{\mathrm{B}}$ objective, and they are the single most important factors affecting feasibility and the objective function value of $T_{\mathrm{A}}$.

In the following, we describe the special observations and techniques we developed to overcome the limitations noted above and transform the indicated decomposition approach into a highly successful solution strategy.

\section{PROPOSITION A}

Given values for $z_{i}, q_{i}$ and $y_{i}$, the $T_{\mathrm{A}}$ model can be formulated as a set of disjoint (one per calendar year) capacitated trans-shipment problems.

The justification of this proposition requires some mathematical manipulation. Before providing a technical exposition, we simply illustrate the form of the resulting capacitated trans-shipment problem and comment on its features. Figure 1 provides such an illustration for a simplified problem in which $T_{\mathrm{A}}$ consists of two time periods, both time periods are in calendar year $T_{k}$, and there is exactly one type of orange product and one type of white product. Constraints (8)-(12) are handled in this diagram as follows. The nodes labeled $S_{i, t}$ denote product type $i$ in period $t$ and those labeled $C_{1 t}$ denote customer 1 in period $t$. The node $\mathrm{O}_{T_{k}}$ represents orange product sold to customer 2 or 3 during calendar year $T_{k}$. Each $S$ node has three arcs directed from it representing flows to customers 1,2 and 3, respectively. The lower and upper bounds on the arcs (denoted by the quantities in parentheses) reflect the impact of the $z_{i}$ and $q_{i}$ variables from the $T_{\mathrm{B}}$ model. The upper bound on the arc from $\mathrm{O}_{T_{k}}$ to $\mathrm{W}_{T_{k}}$ restricts the flow of orange product to customers 2 and 3 , thereby forcing at least half of customer 1's demand to be satisfied by orange product and ensuring no more than half of the demand would be satisfied by white product.

Proof

Clearly, for given values of $z_{i}, q_{i}$, and $y_{i}$, the $T_{\mathrm{A}}$ model separates by calendar year. Constraint (5) couples together period constraints within a calendar year. For a given calendar year $T_{k}$ in $T_{\mathrm{A}}$, constraints (5) can be rewritten, however, as follows:

$$
\sum_{i \in I-I \mathrm{~W}} \sum_{t \in T_{k}}\left(x_{i 2 t}+x_{i 3 t}\right) \leqslant \sum_{i \in I-I \mathrm{~W}} a_{i t} z_{i}-0.5 \sum_{t \in T_{k}} d_{1 t} .
$$




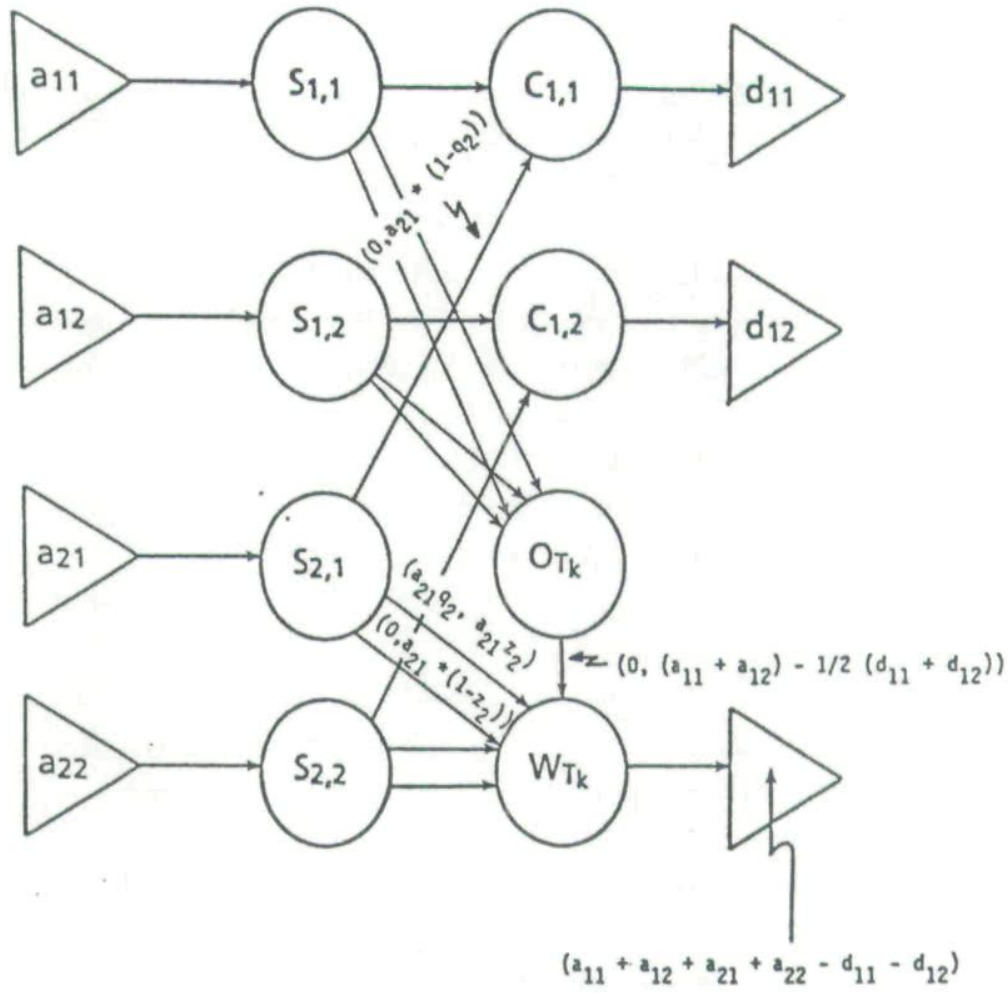

Fig. 1. A network flow representation of a $T_{\mathrm{A}}$ model.

Constraint (5.1) may be replaced by constraints (5.2) and (5.3).

$$
\begin{gathered}
\sum_{i \in I-I \mathrm{~W}} \sum_{t \in T_{k}}\left(x_{i 2 t}+x_{i z t}\right)-y=0 \\
0 \leqslant y \leqslant \sum_{i \in I-I \mathrm{~W}} a_{i t} z_{i}-0.5 \sum_{t \in T_{k}} d_{1 t} .
\end{gathered}
$$

From constraints (8), (9) and (10), form the redundant constraint:

$$
\begin{gathered}
\sum_{t \in T_{k}} \sum_{i \in I} \sum_{j \in J} x_{i j t}-\sum_{t \in T_{k}} \sum_{i \in I} x_{i j t} \\
=\sum_{i \in I_{\mathrm{E}}} a_{i t} y_{i}+\sum_{i \in I_{\mathrm{N}}} a_{i t} y_{i}-\sum_{t \in T_{k}} d_{1 t} .
\end{gathered}
$$


Subtracting (5.2) from (18) yields the redundant constraint:

$$
\sum_{t \in T_{k}} \sum_{i \in I_{\mathrm{W}}}\left(x_{i 2 t}+x_{i 3 t}\right)+y=\sum_{i \in I_{\mathrm{E}}} a_{i t}+\sum_{i \in I_{\mathrm{N}}} a_{i t} y_{i}-\sum_{t \in T_{k}} d_{1 t} .
$$

Replacing constraint (5) with constraints (5.2) and (5.3) and augmenting each calendar year submodel of the $T_{\mathrm{A}}$ model with constraint (19) results in a $T_{\mathrm{A}}$ model composed of a set of disjoint capacitated trans-shipment problems for given values of $z_{i}, q_{i}$, and $y_{i}$.

The major effect of proposition A was to permit the $T_{\mathrm{A}}$ model to be decomposed into a set of six network flow models (one for each calendar year spanning the 61 periods of $T_{\mathrm{A}}$ ), each capable of being solved very quickly by the general purpose network flow code ARCNET. The typical size of each network was 650 nodes and 1900 arcs. The optimization time per network never exceeds one second. ECL made it easy to pass variable values from MPSX to ARCNET, and the general purpose features of ARCNET made it easy to edit the network problem files given these values.

The reformulation of the $T_{\mathrm{A}}$ model to a set of network models and subsequent analysis of the special structure of these networks lead to two additional important insights which are characterized in proposition $\mathrm{B}$ and $\mathrm{C}$ below.

\section{PROPOSITION B}

A set of simple constraints can be generated whose feasible solutions are necessary and sufficient to provide values for $q_{i}$ that are feasible to the network flow representation of the $T_{\mathrm{A}}$ model.

Proof

Consideration of the network flow problem illustrated in fig. 1 discloses that $q_{i}$ must satisfy the following conditions for there to be any feasible solution to the $T_{\mathrm{A}}$ model:

$$
\sum_{i \in I} a_{i t} q_{i} \leqslant \sum_{i \in I_{\mathrm{E}}} a_{i t}+\sum_{i \in I_{\mathrm{N}}} a_{i t} y_{i}-d_{1 t} \quad \text { for each } t \in T_{\mathrm{A}} .
$$

Were constraints (20) not satisfied, there would be insufficient supply available to meet customer 1's demand in each period.

The network formulation also implies that the $q_{i}$ must satisfy the following constraints for there to be any feasible solution to the $T_{\mathrm{A}}$ model: 


$$
\begin{aligned}
\sum_{t \in T_{k}} \sum_{i \in I_{\mathrm{O}}} a_{i t} q_{i} & \leqslant \sum_{t \in T_{k}}\left(\sum_{i \in I_{\mathrm{O}} \cap I_{\mathrm{E}}} a_{i t}+\sum_{i \in I_{\mathrm{O}} \cap I_{\mathrm{N}}} a_{i t} y_{i}\right) \\
& -\sum_{t \in T_{k}} 0.5 d_{1 t}
\end{aligned}
$$

for all $k$ such that $T_{k}$ in $T_{\mathrm{A}}$ :

Here, $I_{\mathrm{O}}$ denotes the set of product types belonging to the orange class. These constraints ensure that the restrictions governing the percentage of orange product which must go to customer 1 on a calendar year basis are met. They are based upon the conservation of flow through node $\mathrm{O}_{T_{k}}$ in the network diagram. That is, since customer 1 can receive no more than one half of his demand in white product, he must receive at least half of it in orange. This, in tum, bounds the amount of orange product that can go to customers 2 and 3 . The sufficiency of these constraints to provide feasible network solutions follows from the fact that there is only one specified demand per time period in the $T_{\mathrm{A}}$ model. Constraint (20) ensures that there is sufficient product to meet this demand and constraint (21) ensures that the orange product percentage can be accommodated while meeting the requirement that total demand equal total supply.

Constraints (20) and (21), which numbered about 24 in the actual problem, were added to model $T_{\mathrm{B}}$ to ensure feasibility for approximately 4000 constraints in model $T_{\mathrm{A}}$.

It was assumed earlier that no constraint (5) included variables from both $t \in T_{\mathrm{B}}$ and $t \in T_{\mathrm{A}}$ (or in other words, that $T_{\mathrm{B}}$ ended at the end of a calendar year and $T_{\mathrm{A}}$ began at the start of the subsequent calendar year). In fact, this was not the case. For exactly one $k, T_{k}$ partitioned into two subsets $T_{k} \cap T_{\mathrm{B}}$ and $T_{k} \cap T_{\mathrm{A}}$. To effect the decomposition, it was necessary, for that $k$ only, to change constraints (5) in model $T_{\mathrm{B}}$ to:

$$
\sum_{i \in I_{\mathrm{O}}} \sum_{t \in T_{k} \cap T_{\mathrm{B}}} x_{i 1 t}+u_{k}=0.5 \sum_{t \in T_{k}} d_{1 t} \text {, }
$$

where $u_{k}$ is an unrestricted variable, and for this $k$ to change constraint (21) to

$$
\sum_{t \in T_{k} \cap T_{\mathrm{A}}} \sum_{i \in I_{\mathrm{O}}} a_{i t} q_{i} \leqslant \sum_{t \in T_{k} \cap T_{\mathrm{A}}}\left(\sum_{i \in I_{\mathrm{O}} \cap I_{\mathrm{E}}} a_{i t}+\sum_{i \in I_{\mathrm{O}} \cap I_{\mathrm{N}}} a_{i t} y_{i}\right)-u_{k} .
$$

The variable $u_{k}$ measures the extent to which the restriction that no more than onehalf of the demand during a calendar year be met with orange product is satisfied by flows in $T_{\mathrm{B}}$ so that flows in $T_{\mathrm{A}}$ must compensate to ensure the constraint is met. 


\section{PROPOSITION C}

Penalty coefficients for the $q_{i}$ variables to incorporate into the $T_{\mathrm{B}}$ objective can be deduced from the network flow model.

This proposition can be demonstrated straightforwardly as follows. Since customer 3 pays more for product than customer 2 , the solution to the network form of the $T_{\mathrm{A}}$ model with all $z_{i}=q_{i}=0$ represents an upper bound $C$ on the revenues that could be obtained from sales during $T_{\mathrm{A}}$. Moreover, as $q_{i}$ increases from 0 , the effect on the solution of the $T_{\mathrm{A}}$ model is that flow on the arc corresponding to sales to customer 3 will move to the arc corresponding to sales to customer 2 . The impact on the model $T_{\mathrm{A}}$ objective is a change of at least $\Sigma_{t \in T_{\mathrm{A}}} a_{i t}\left(C_{i 3 t}-C_{i 2 t}\right) q_{i}$. Hence $\Sigma_{t \in T_{\mathrm{A}}} a_{i t}\left(C_{i 3 t}-C_{i 2 t}\right)$ is a legitimate penalty to attach to $q_{i}$ in the $T_{-\mathrm{B}}$ model objective. Coupling the use of this penalty with adding the constant $C$ to the $T_{\mathrm{B}}$ model objective produces an optimistic estimator of the sum of the $T_{\mathrm{A}}$ and $T_{\mathrm{B}}$ objectives.

Propositions $\mathrm{A}, \mathrm{B}$ and $\mathrm{C}$, in combination, make the decomposition approach a viable solution strategy. Its effectiveness derives from the following principal reasons:

(a) Model $T_{\mathrm{A}}$ is very easy to solve.

(b) The solution of a suitable linear relaxation of $T_{\mathrm{B}}$ by itself yields a strong bound in a branch and bound scheme.

(c) The segregation of the $T_{\mathrm{A}}$ and $T_{\mathrm{B}}$ components provides a strong problem representation because feasibility to the constraints of model $T_{\mathrm{A}}$ are incorporated in constraints (20) and (21) and made part of model $T_{\mathrm{B}}$.

To provide a complete solution algorithm based on this decomposition, it was critical that some means be found to solve a linear relaxation of model $T_{\mathrm{B}}$ effectively. The chief requirement is an effective mechanism for handling the nonlinear constraints of (7).

The last major key to the success of the decomposition is the following:

\section{NONLINEAR CONSTRAINT STRATEGY}

Constraints (7) are replaced alternately by linear constraints which underestimate and overestimate the true value of each $q_{i}$.

An underestimating value for each $q_{i}$ is easy to achieve. For the purpose of computing such an estimate, the constraints of (7) may clearly be replaced by

$$
\frac{\sum_{t \in T_{\mathrm{c}}} x_{i 2 t}}{\sum_{t \in T_{\mathrm{c}}} a_{i t}}=q_{i} .
$$


For the purpose of finding feasible solutions to $T_{\mathrm{B}}$, two possibilities exist:

(a) Constraint (7) could be replaced by (7u) and the LP relaxation of model $T_{\mathrm{B}}$ solved. The true value of each $q_{i}$ could be computed using the underestimating solution, using the values of the $x_{i 2 t}$ found using (7u) in the expression for $q_{i}$ in (7). If these values are feasible to constraints (20) and (21) and if the $y_{i}$ and $z_{i}$ are integer (or rounded), then a candidate incumbent solution can be determined for the problem as a whole by re-solving model $T_{\mathrm{B}}$ with $q_{i}$ fixed at the true values, $z_{i}$ and $y_{i}$ fixed, and solving model $T_{\mathrm{A}}$ with the same values of $q_{i}, z_{i}$ and $y_{i}$.

(b) The LP relaxation of model $T_{\mathrm{B}}$ can be solved with constraint (7) replaced by

$$
\frac{\sum_{t \in T_{\mathrm{c}}}\left(x_{i 2 t}+x_{i 3 t}\right)}{\sum_{t \in T_{\mathrm{c}}} a_{i t}}=q_{i},
$$

which clearly overestimates the true value of $q_{i}$. If the LP relaxation of model $T_{\mathrm{B}}$ is feasible, then the true $q_{i}$ corresponding to the overestimate of $q_{i}$ are feasible to model $T_{\mathrm{A}}$ because constraints (20) and (21) are satisfied by the unnecessarily large values of the $q_{i}$. Further, all of the $z_{i}$ and $y_{i}$ can be rounded to yield a feasible solution to model $T_{\mathrm{B}}$. Thus, a candidate incumbent solution can be generated as described above.

\section{Computational implementation of the decomposition algorithm}

Implementation of the algorithm was a challenging task because of the variety of optimization problems that might be solved at any node of the branch and bound tree and the attendant 'housekeeping' that was required. For example, to obtain the constant $C$, the network flow model $T_{\mathrm{A}}$ had to be solved with all $z_{i}=q_{i}=0$. When any incumbent was to be generated, the network flow model had to be edited to reflect the appropriate values of $z_{i}$ and $q_{i}$ in the lower and upper bounds associated with an arc. Further, several LP relaxations of model $T_{\mathrm{B}}$ might need to be solved at a node. These included forms with penalty coefficients on the $q_{i}$ and either the underor overestimating constraints (7o) or $(7 \mathrm{u})$ active, and others with 0 coefficients on the $q_{i}$ variables and each $q_{i}$ assigned a fixed value in constraints (7).

Thus, there needed to be routines to perform such edits on the LP matrix as fixing variable bounds, activating or deactivating rows and changing objective function coefficients 'on the fly' during the branch and bound. To expedite the solution process, optimal bases needed to be saved and restored for the overestimating, underestimating or incumbent defining forms of the problem. Finally, to permit restart capability in case of a system crash during an extended run, it was desirable for the 
program to perform periodic array dumps. This same capability enabled users to solve a problem in several computer runs, to review the progress made in the latest run, or to change parameters (such as the increasing optimality tolerance) between runs.

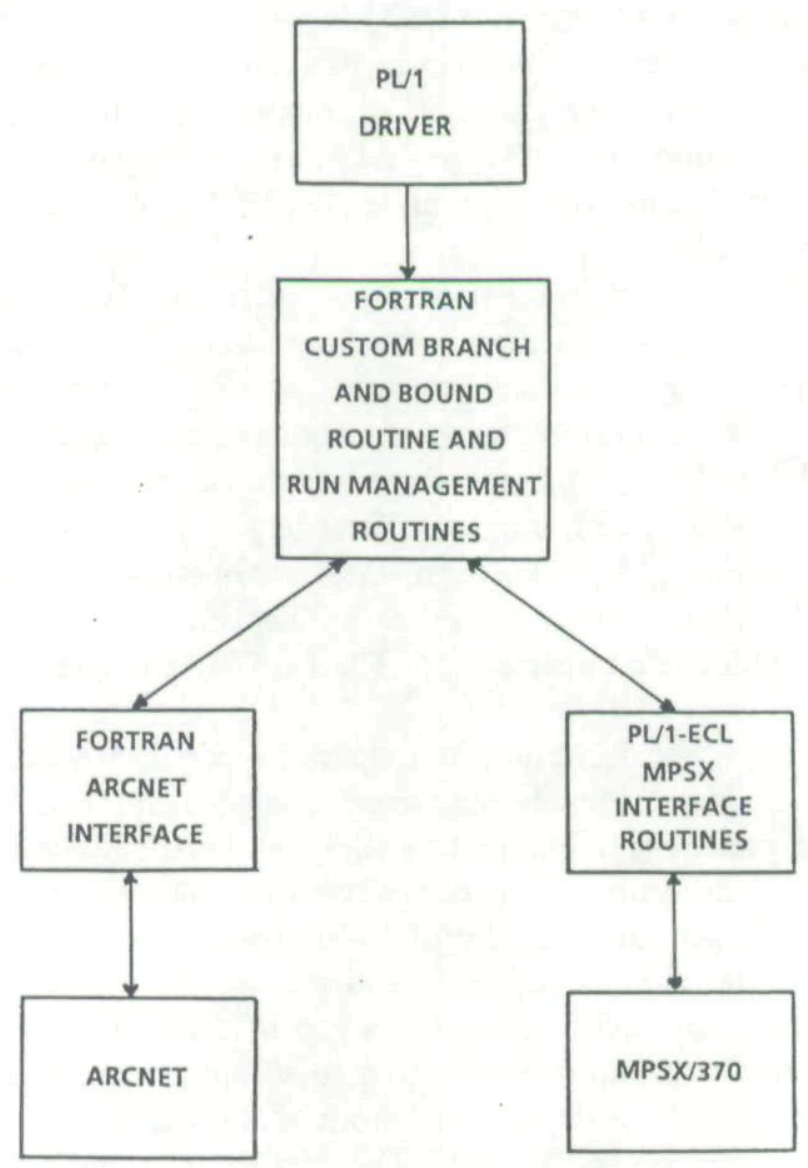

Fig. 2. Principal software components and interrelationships.

The major components of the system and their interrelationships are represented in fig. 2. This design exploits the capability of PL/1 to interface simultaneously with FORTRAN and with MPSX (by the way of the extended control language feature of MPSX). First, a PL/1 main program was written for the sole purpose of initiating the FORTRAN branch and bound routine that was the heart of the system. The FORTRAN program was responsible for the usual branch and bound tasks of maintaining the candidate list, selecting separation variables, and maintaining a list of 
incumbents. Further, it ascertained from the model solution and the stage of the algorithm what problem edits were necessary. Second, the retrieval of solutions and the edits to problems were carried out via special purpose routines written in PL/1 ECL. Optimal solutions could be passed from MPSX arrays to the FORTRAN program and lists of parameters, variables or structural components and how they should be changed could be passed via arrays to MPSX. In all, some sixteen P/L1 routines were used. Third, similar routines were written in FORTRAN to enable the network flow problems to be edited and to pass solution information back to the branch and bound routine. Lastly, two optimizers, IBM's general linear programming system MPSX/370 and ARC's primal simplex network optimizer ARCNET, provided the solution power on which the decomposition rested.

Initial implementation required three calendar months. Refinements, mostly to the branching strategy, continued on a part-time basis over three additional months. The branching strategy that worked best gave priority to those $q_{i}$ for which the difference between the underestimate and the actual value $q_{i}$ was greatest. The degree of error weighted by the penalty coefficient (defined by proposition $\mathrm{C}$ ) was the deciding factor, so that a $q_{i}$ with large error and large penalty would lead to a separation based upon setting $q_{i}=0$. Through input parameters, the branch and bound routine could be directed to select candidate problems in LIFO order or based upon the value of each candidate's upper bound. The LIFO strategy reliably outperformed the alternative.

By all measures, the decomposition algorithm performed extremely well. The overestimating constraints (7o) were instrumental in identifying an incumbent at the initial node of the branch and bound tree that was invariably within $12 \%-17 \%$ of the upper bound on the optimum objective function value determined at that stage of the solution. In longer runs (usually of 1000 nodes or less), the gap between the best incumbent and the optimum upper bound was usually reduced to $7 \%$ and often to $5 \%$ or less. A $95 \%$ optimality level (i.e. a gap of $5 \%$ ) was used as a termination criterion. Several unique scenarios were solved to within $1 \%$ or $2 \%$ of optimality and required very few nodes. Run times varied from as short as a few cpu minutes to as long as fourteen cpu hours on an IBM 3033. The time to solve the initial linear program was typically around six cpu minutes and reoptimization after a branch took around forty cpu seconds. ARCNET required consistently 3-4 cpu seconds in total to solve all of the networks that comprised model $T_{\mathrm{A}}$. Large numbers of incumbents were generated in every run, and a list of the five best incumbents encountered was maintained by the branch and bound routine. This experience documents that the combination of off-the-shelf optimizers, sophisticated interfaces like ECL and customized algorithms can be very effective and very powerful.

Computer graphics played an important role in reporting solutions and summarizing input data. As mentioned earlier, the persons responsible for formulating strategies by hand for fulfilling contract terms had been using two graphs to develop their plans. When the first solution based on a mathematical model was plotted in the 
same format, it was immediately apparent that the model had found a markedly better solution. This contributed greatly to the credibility of a modeling approach because the users could literally see the improvements. The graphical representation was extremely powerful for validating solutions and for comparing solutions to different scenarios. Once, early in the project, an error in a right-hand side coefficient led the model to generate a solution that violated contract terms and this was readily apparent on one of the graphs. That a solution to a 12000 variable 8000 row mathematical programming problem can be so well communicated by two graphs speaks highly for the power and importance of graphics in presenting complex results in a friendly comprehensible fashion.

\section{Summary}

This paper documents the power that mathematical programming based systems offer management in developing good solutions to difficult, substantive problems. The stakes in this application justified the effort to build a special purpose algorithm and software system for solving the problem. Clearly, building blocks exist which can be used readily to develop valuable decision analytical systems.

\section{References}

[1] ARCNET User's Manual (Analysis, Research and Computation, Inc., Austin, Texas, 1981).

[2] E.M.L. Beale, Mathematical Programming in Practice (Pitman, 1968).

[3] D.M. Himmelblau, Applied Nonlinear Programming (McGraw-Hill, New York, 1972).

[4] IBM Mathematical Programming System Extended/370 (MPSX/370) Program Reference Manual, 3rd Edition (International Business Machines Corporation Technical Publications Department, White Plains, NY, 1978).

[5] L. Lasdon and A. Waren, Survey of nonlinear programming applications, Oper. Res. 28 (1980)1029.

[6] F. Palacios-Gomez, L. Lasdon and M. Engquist, Nonlinear optimization by successive linear programming, Management Science 28(October, 1982). 
Copyright of Annals of Operations Research is the property of Springer Science \& Business Media B.V.. The copyright in an individual article may be maintained by the author in certain cases. Content may not be copied or emailed to multiple sites or posted to a listserv without the copyright holder's express written permission. However, users may print, download, or email articles for individual use. 